\title{
Erratum to: Investigation on the Flexural Creep Stiffness Behavior of PC-ABS Material Processed by Fused Deposition Modeling Using Response Surface Definitive Screening Design
}

\author{
OMAR AHMED MOHAMED $®{ }^{1,3}$ SYED HASAN MASOOD, ${ }^{1}$ \\ and JAHAR LAL BHOWMIK ${ }^{2}$
}

1.-Department of Mechanical and Product Design Engineering, Swinburne University of Technology, Hawthorn, VIC, Australia. 2.-Department of Statistics, Data Science and Epidemiology, Swinburne University of Technology, Hawthorn, VIC, Australia. 3.-e-mail: Omar.Ahmed. Mohamed@outlook.com

\section{Erratum to: JOM}

\section{DOI: 10.1007/s11837-016-2228-z}

This article has been updated to correct errors introduced during the production process. The \pm in Eq. 2 has been corrected.

$$
\begin{aligned}
\operatorname{FCS}(\mathrm{MPa})= & 902.487-29.387 \times\left[\frac{\left(x_{1}-0.2540\right)}{0.0762}\right]-250.631 \times\left[\frac{\left(x_{2}-0.25\right)}{0.25}\right]+28.794 \times\left[\frac{\left(x_{3}-45\right)}{45}\right] \\
& -13.417 \times\left[\frac{\left(x_{4}-45\right)}{45}\right]-8.331 \times\left[\frac{\left(x_{5}-0.5177\right)}{0.0605}\right]+218.064 \times\left[\frac{\left(x_{6}-5\right)}{5}\right] \\
& +\left[\frac{\left(x_{1}-0.2540\right)}{0.0762}\right] \times\left\{\left[\frac{\left(x_{3}-45\right)}{45}\right] \times 59.672\right\}+\left[\frac{\left(x_{4}-45\right)}{45}\right] \times\left\{\left[\frac{\left(x_{4}-45\right)}{45}\right] \times 30.083\right\} \\
& +\left[\frac{\left(x_{2}-0.25\right)}{0.25}\right] \times\left\{\left[\frac{\left(x_{6}-5\right)}{5}\right] \times 232.638\right\}+\left[\frac{\left(x_{5}-0.5177\right)}{0.0605}\right] \times\left\{\left[\frac{\left(x_{6}-5\right)}{5}\right] \times 67.608\right\}
\end{aligned}
$$

